\title{
Surface enhanced Raman scattering substrate based on gold-coated anodic porous alumina template
}

\author{
G. Das ${ }^{a, *}$, N. Patra ${ }^{a, b}$, A. Gopalakrishanan ${ }^{a, b}$, R. Proietti Zaccaria ${ }^{a}$, A. Toma ${ }^{a}$, S. Thorat ${ }^{a, b}$, E. Di Fabrizio ${ }^{\text {a }}$, \\ A. Diaspro ${ }^{a}$, M. Salerno ${ }^{a}$ \\ a Nanostructures, Istituto Italiano di Tecnologia, via Morego, 30, 16163 Genova, Italy \\ ${ }^{\mathrm{b}}$ Università degli Studi di Genova, Genova, 16145, Italy
}

\section{A R T I C L E I N F O}

\section{Article history:}

Available online 19 March 2012

\section{Keywords:}

SERS

Large area SERS substrate

Cresyl violet

Anodic porous alumina

\begin{abstract}
A B S T R A C T
We report the fabrication of large area SERS substrates based on anodic porous alumina template over which gold thin layer of $25 \mathrm{~nm}$ was deposited. A specific design of honey-comb structure with the pore size/cell thickness of 60/40 nm was attained by using the controlled electrolyte potential, time of etching and oxalic acid as an electrolytic solution. Cresyl violet and glycine molecules were deposited through chemisorption technique to verify the SERS substrate functionality. Numerical simulations in order to describe the realistic device were also carried out, finding consistency with the experimental results.
\end{abstract}

(c) 2012 Elsevier B.V. All rights reserved.

\section{Introduction}

In last few years, plasmonics based sensor device such as surface enhanced Raman spectroscopy (SERS) has attracted lots of attention to the researchers. When an electromagnetic light interacts with a molecule situated in the vicinity of nano-metal surface, due to the collective oscillation of conduction electrons, an enhanced electric field is observed. The Raman scattering observation with this enhanced electric field close to metal surface leads to an enhanced Raman scattering or in other way can be called as surface enhanced Raman scattering [1,2]. There are many techniques such as electron beam lithography (EBL) $[3,4]$, optical lithography with reactive ion etching [5], focused ion beam (FIB) [6], colloidal technique [7-9], metal island film [10], etc. by which SERS substrates can be fabricated. There is always a competition regarding the quality of the nanostructure, fabrication area, time and cost. EBL is the most efficient technique to reproduce the nano-patterned SERS substrates with high spatial precision $[11,12]$ but faces critical limitations besides many advantages regarding large area fabrication whereas colloidal technique is well known for large area metal deposition but showing minimum reproducibility. Indeed, there are critical challenges to resolve which impede the SERS applications: how to increase significantly the average-area of SERS enhancement and how to overcome with the sparse distribution of hot-spots problem. In the past, Zhang et al. $[13,14]$ reported a new technique called nanosphere lithography in which shadow evaporation through self-assembled arrays of polystyrene nanosphere was used to

\footnotetext{
* Corresponding author.

E-mail address: gobind.das@iit.it (G. Das).
}

fabricate a large area SERS substrate. Herein, we propose a new way to fabricate cheap and high enhancement SERS substrate based on anodic porous alumina (APA) which could be a trade-off between the two above limits such as large fabrication area and average-area SERS enhancement factor.

In the present work, APA templates with hexagonal periodicity [15] were fabricated, as reported in literatures [16-18]. A thin layer $(25 \mathrm{~nm})$ of gold was then deposited onto APA substrates with the purpose to obtain nano-patterned metal surface. Using this technique, we are able to achieve reproducible SERS substrates with wall thickness and pore diameter down to 40 and $60 \mathrm{~nm}$, respectively. This structures show very efficient SERS signal even in the presence of fluorescent molecules. Therefore, the substrate behaves as an efficient fluorescence quenching device, as already shown for metallic nano-particles [19]. The surface morphology was characterized by scanning electron microscopy (SEM), and was probed with cresyl violet (CV) and glycine amino acid. Numerical simulations were also performed in order to support the experimental findings.

\section{Experimental}

In this work, APA substrate with hexagonal periodicity [15] was prepared at $+7^{\circ} \mathrm{C}$ external bath temperature with electrolyte stirring, by controlled modification of a $250 \mu \mathrm{m}$ thick foil of ultrapure (99.999\%) aluminum as anode, in the same open electrochemical cell configuration as in previous experiments [20]. The anodization was carried out in the aqueous solution of oxalic acid in order to obtain the desired pore size and cell thickness. The approximate design of the nanostructured APA template is 

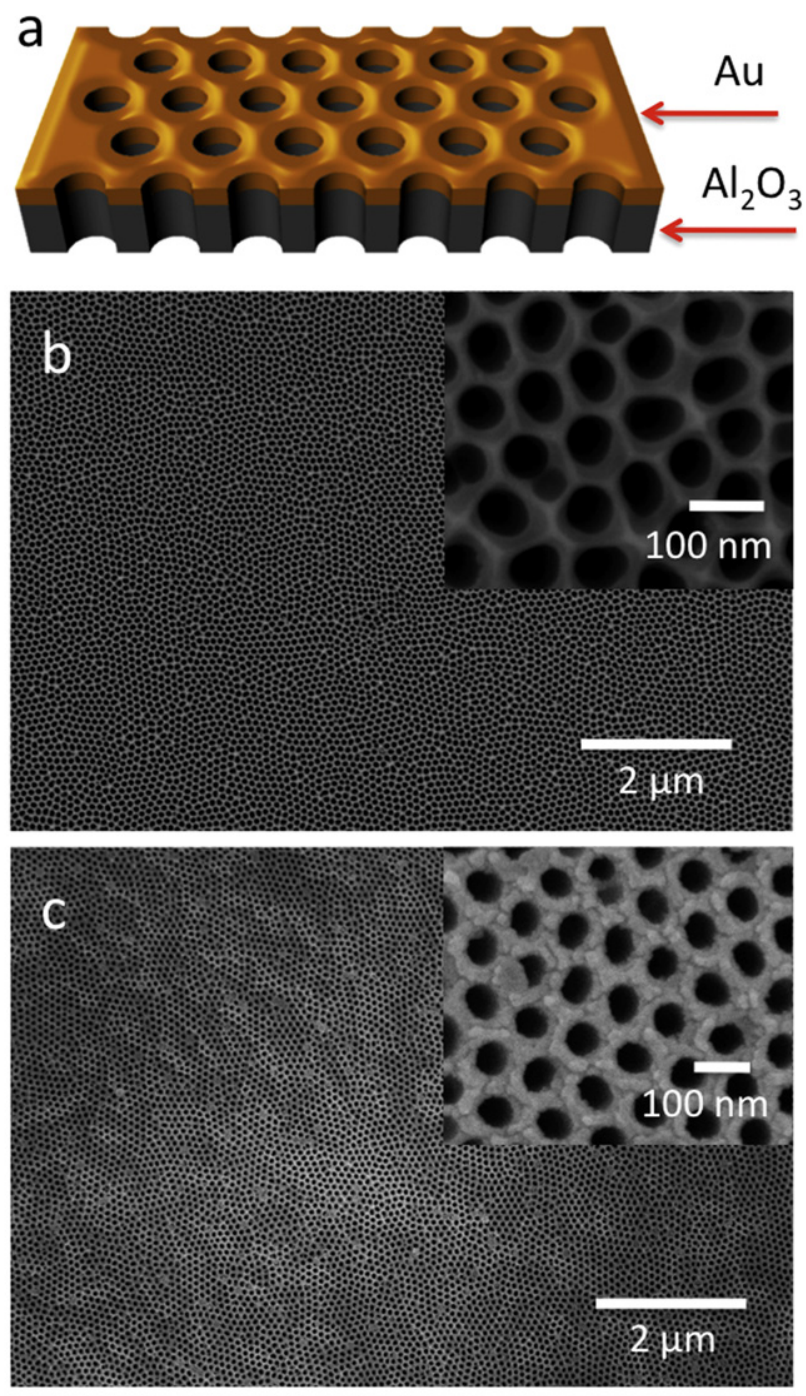

Fig. 1. (a) Illustration of honey-comb structure; SEM images of anodic porous alumina substrate before and after gold-coating of $25 \mathrm{~nm}$ are shown in figures (b) and (c), respectively.

illustrated in Fig. 1a. To be noted, the electrolytic potential, the time of etching and the acid concentration should be chosen very carefully in order to have a specific pore size/cell thickness.

Gold was thermally evaporated onto the APA substrates (APA) with deposition rate of $0.5 \AA / s$ until a final total thickness of $\sim 25 \mathrm{~nm}$ was reached. The sample holder was always rotated at 1 RPM in order to improve the uniformity and the morphological quality of the gold layer. The substance of interest was deposited over resulting gold-coated APA substrate (termed 'AuAPA') using chemisorption technique. In this process, the substrate was dipped in a solution containing the molecule of interest. The substrate was removed after $20 \mathrm{~min}$ from the solution and then, gently, rinsed to remove excess molecules those are not attached directly to the metal surface. Thereafter, the samples were dried in $\mathrm{N}_{2}$ flow, and finally stored in a desiccator before SERS measurements. Cresyl violet $(\mathrm{CV})$ dyes and glycine aminoacid were tested for SERS probes with the concentration in the order of $10^{-6} \mathrm{M}$.

\subsection{Topographic measurement}

SEM measurements (JSM-7500FA, JEOL, Japan) were performed on the APA substrates, keeping the $15 \mathrm{kV}$ of acceleration voltage for primary electron beam and collecting the topographic signal from the secondary electrons. SEM images before and after gold deposition are shown in Fig. 1b and c. From SEM image, the pore size and cell thickness were found to be 60 and $40 \mathrm{~nm}$, for gold coated SERS substrate.

\subsection{Optical measurements}

Optical absorption measurement of the molecule was carried out in quartz cuvette by means of Varian (model: Cary 6000) UV-VIS-NIR double spectrometer in the range of $200-800 \mathrm{~nm}$.

Micro-probe SERS measurements were performed using Renishaw inVia Raman microscopy. The equipment consists of excitation laser $(633 \mathrm{~nm})$, grating with 1800 lines/mm and microscopy objective 100X (NA: 0.9)in backscattering geometry. The measurements were performed with laser power $5.5-78 \mu \mathrm{W}$ and with integration time $20-50 \mathrm{~s}$.

\subsection{Simulation for electric field distribution}

In order to provide the theoretical support regarding electrical field distribution which leads to an increase in absolute electric field, numerical simulations by means of CST Microwave studio were executed. In this analysis, tetragonal mesh was used. The nanostructured design is kept to be as close as the experimental one.

\section{Results and discussion}

Cresyl Violet dye (see optical absorption spectrum and molecular structure in inset of Fig. 2a is extensively used in biology and medicine for histological stain. The optical spectrum of CV shows the absorption peak at around $580 \mathrm{~nm}$. Though it is not fully in resonance with the excitation frequency for SERS measurement, minute resonance contribution can be expected due to the resonance frequency. SERS measurements were performed for CV dye, deposited on AuAPA SERS substrate, at different positions in the range of $300-1400 \mathrm{~cm}^{-1}$. Raman measurements were performed for CV on a flat non-patterned silicon wafer substrate and on flat gold surface as well, which can be considered as a positive control sample. The Raman spectrum of CV on Si surface, presented in Fig. 2a, is relatively featureless, with an exponentially increasing fluorescence background and a single characteristic peak of CV at $\sim 591 \mathrm{~cm}^{-1}$ with low intensity.

AuAPA SERS substrate background measurement without any molecule over the surface was carried out, shown in the inset of Fig. 2b. Figure shows a flat background response without any Raman features through-out the spectral range, indicating a SERS substrate without any kind of impurity over it.

SERS measurements were accomplished on CV molecule, deposited onto AuAPA substrates with pore diameter $(\sim 60 \mathrm{~nm})$ and wall thickness ( $40 \mathrm{~nm}$ ) (Fig. 2b. The characteristic vibrational bands of CV are observed [21], centered at around 348, 591, 675 and $1186 \mathrm{~cm}^{-1}$, can be attributed to the out of plane skeleton deformation, combination of in-plane $\mathrm{N}-\mathrm{H}_{2}$ and ring bending, ring deformation and combination of $\mathrm{N}-\mathrm{H}_{2}$ rocking and $\mathrm{C}-\mathrm{H}_{\mathrm{x}}$ rocking, respectively [22]. From the vibrational bands observed in the Fig. 2b, it is most probable that the CV is oriented in such a way that the $-\mathrm{N}-\mathrm{H}_{2}$ group is closer to the gold film, causing enhanced scattering and, consequently, higher SERS signal. Additionally, in Fig. 2b, the high exponential background appeared in Fig. 2a for $\mathrm{CV}$ on silicon substrate was disappeared. Hence, significant fluorescence quenching is also illustrated, as already observed in the past for gold nanoparticles [19]. Furthermore, the positive control measurements of $\mathrm{CV}$, deposited on flat gold surface were also conducted, shown in inset of Fig. 2. It is noticeable that though 

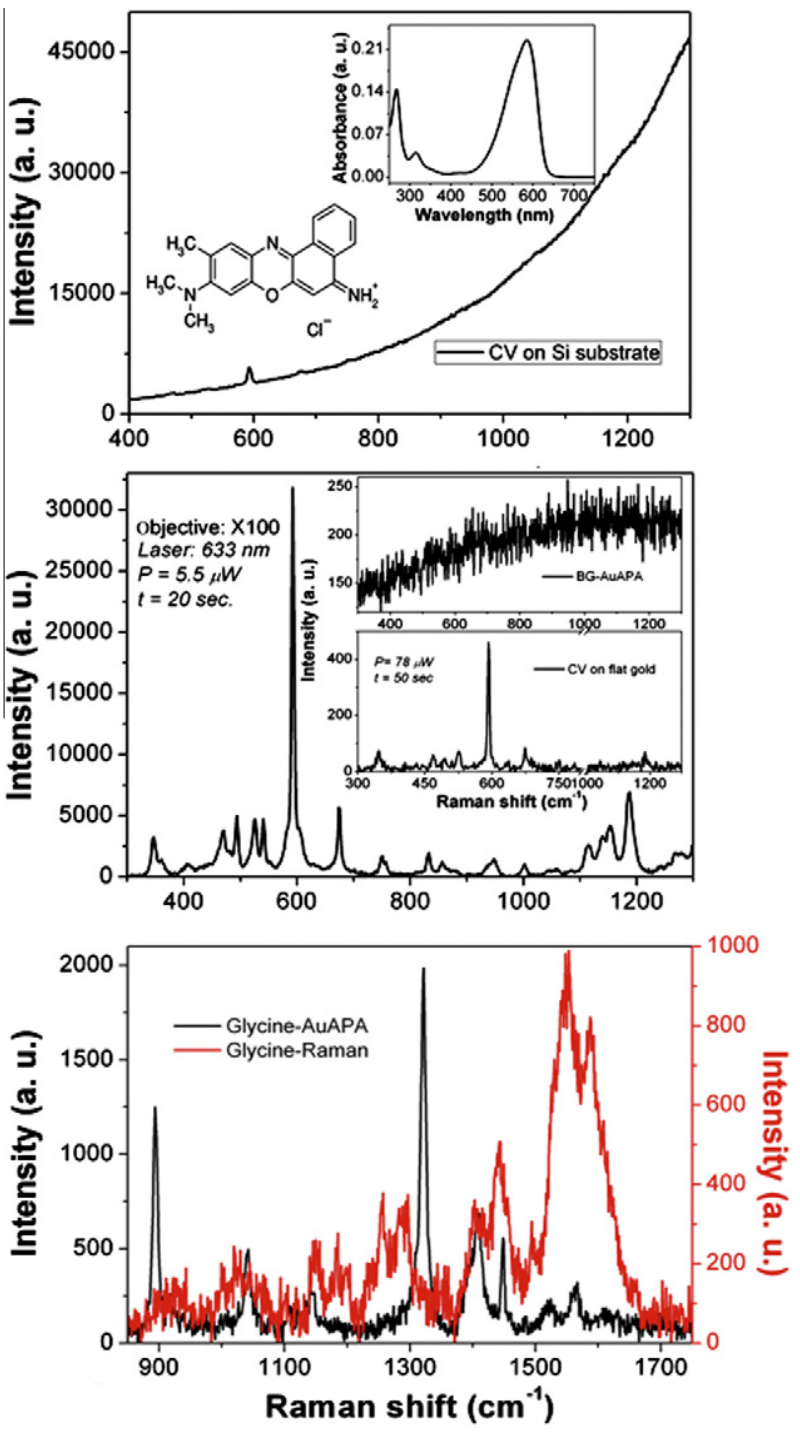

Fig. 2. (a) Raman spectrum of cresyl violet deposited on Si substrate, showing high fluorescence background in the range of 400-1550. Molecular structure and optical absorption spectrum of CV are shown in the inset of the same figure (b) SERS spectrum of $C V$ is also illustrated, showing the CV spectrum due to an efficient fluorescence quenching by honey-comb structure. AuAPA SERS background spectrum and Raman spectrum of CV, deposited on flat Au surface, are shown in the inset of figure (c) SERS and Raman spectrum of glycin (an amino acid).

the laser power and integration time are increased, the intensity of $\mathrm{CV}$ at $591 \mathrm{~cm}^{-1}$ is found very weak. Considering that the CV molecules are only deposited onto the AuAPA cell wall and taking into account the power, the integration time and the peak height of $591 \mathrm{~cm}^{-1}$ band from Fig. 2, the SERS enhancement factor AuAPA device is estimated to around $10^{4}$ and $7 \times 10^{6}$ with respect to the flat gold surface and to the Si substrate, respectively (see Supplementary info section 1 for calculation).

Various measurements (around 100 spectra) were carried out on this SERS AuAPA substrate at different points, giving identical Raman vibrational frequency of $\mathrm{CV}$. It shows the equal averagearea enhancement factor and also the reproducible spectral/substrate behavior at different locations of the SERS device.

In addition, glycine, an amino acid, was also tested over AuAPA substrate. Fig. 2c shows the glycine SERS and Raman spectra in the range of $850-1750 \mathrm{~cm}^{-1}$. Various chemical vibrational bands are observed centered at around 891 (C-C stretching), 1041 (C-N stretching), 1321 ( $\mathrm{CH}_{2}$ wagging), 1410 (COO- symmetric stretching), and 1450 ( $\mathrm{C}-\mathrm{H}_{2}$ bending $\mathrm{cm}^{-1}$. Various bands but in lower (a)

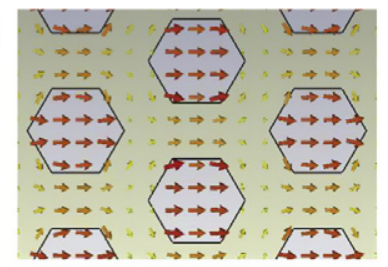

(b)

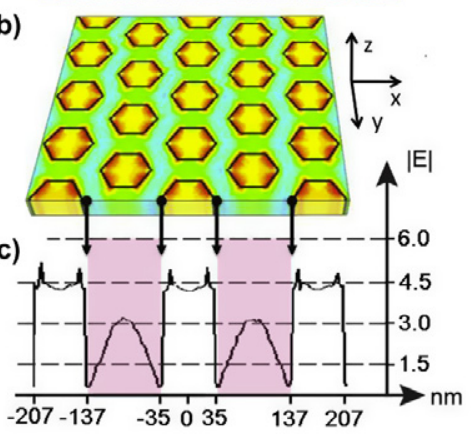

Fig. 3. (a) Vectorial distribution of the electric field on honey-comb structure. (b) The overall electric field distribution in $\mathrm{x}-\mathrm{y}$ plane for incident light polarized along $\mathrm{x}$-direction; (c) absolute value of the electric field profile along the highlighted horizontal line. The excitation light is $\lambda=633 \mathrm{~nm}$ on gold surface with dielectric function $\varepsilon_{\text {real }}=9.83$ and $\varepsilon_{\text {imaginary }}=1.97$.

intensity are also observed in the range of $1500-1610 \mathrm{~cm}^{-1}$ which are principally related to the $\mathrm{N}-\mathrm{H}_{2}$ deformation stretching vibration $[23,24]$. However, SERS spectrum of glycine, deposited over AuAPA, shows the widening in chemical vibrational bands. The major difference can be observed regarding the band intensity related to the $\mathrm{N}-\mathrm{H}_{2}$ vibrations which most probably is attached to the metal surface in such a way that a significant increase in intensity can be observed.

Near-field electric distribution was calculated by means of the commercial numerical software CST. The motivation was to support the experimental results from SERS substrates. In particular, a gold film with thickness $\sim 25 \mathrm{~nm}$, showing a hexagonal periodic pattern of $100 \mathrm{~nm}$, was simulated. Air-pores with diameter of $60 \mathrm{~nm}$ were designed and the structures were illuminated with an excitation source of $633 \mathrm{~nm}$, polarized along x-direction [25]. In Fig. 3a, we report the vectorial electric field distribution which appears to be localized mainly in the pore-edge regions. The $\mathrm{x}$ polarization creates anti-phase hot spots along the x-axis which determines the abrupt change of the electric field, shown in Fig. 3b. The profiles, shown in Fig. 3c, give even a better image of this behavior.

\section{Conclusions}

Summing up, a new feasible way to produce large area SERS devices was, herein, demonstrated. In details, we have fabricated AuAPA substrates showing periodic hexagonal lattice $(100 \mathrm{~nm})$ with pore size of $60 \mathrm{~nm}$. Our procedure allows the production of reproducible SERS substrates without involving cost effective lithographic approaches. SERS analysis was performed for CV and glycine molecules, deposited by means of chemisorption techniques. The enhancement factor ' $G$ ' was estimated to be around $10^{4}$ and $7 \times 10^{6}$ with respect to the flat gold surface and to the Si substrate, respectively. Numerical simulations of AuAPA device were performed showing a maximum value of electric fields in correspondence of the edges between gold and air-pores. Furthermore, the electric field enhancement is supporting experimental findings.

Systematic study should be carried out by varying pore size and cell thickness. In this way, an optimization of ' $G$ ' could be achieved, opening a new route for the production of disposable SERS device. 


\section{Acknowledgements}

This work has been supported by European Projects SMD FP7-NMP 2800-SMALL-2 (proposal No. CP-FP 229375-2), NANOANTENNA FP7-HEALTH-2009 (Grant Agreement No. 241818) and FOCUS FP7 No. 270483.

\section{Appendix A. Supplementary data}

Supplementary data associated with this article can be found, in the online version, at doi:10.1016/j.mee.2012.02.037.

\section{References}

[1] C.L. Haynes, A.D. McFarland, R.P. Van Duyne, Anal. Chem. 77 (2005) 338A$346 \mathrm{~A}$

[2] R. Jin, Angew. Chem. Int. Ed. 49 (2010) 2826-2829.

[3] G. Das, F. Mecarini, F. Gentile, F. De Angelis, M.H.G. Kumar, P. Candeloro, C. Liberale, G. Cuda, E. Di Fabrizio, Biosens. Bioelectron. 24 (2009) 1693-1699.

[4] M. Kahl, E. Voges, S. Kostrewa, C. Viets, W. Hill, Sens. Actuators B: Chem. 51 (1998) 285-291.

[5] F. De Angelis, F. Gentile, F. Mecarini, G. Das, M. Moretti, P. Candeloro, G. Cojoc, A. Accardo, C. Liberale, R.P. Zaccaria, G. Perozziello, L. Tirinato, A. Toma, G. Cuda, R. Cingolani, E. Di Fabrizio, Nat. Photonics 5 (2011) 682-687.

[6] S. Cabrini, A. Carpentiero, R. Kumar, L. Businaro, P. Candeloro, M. Prasciolu, A. Gosparini, C. Andreani, M. De Vittorio, T. Stomeo, E. Di Fabrizio, Microelect. Eng. 78-79 (2005) 11-15.
[7] K. Kneipp, H. Kneipp, J. Kneipp, Acc. Chem. Res. 39 (2006) 443-450.

[8] S. Nie, S.R. Emory, Science 275 (1997) 1102-1106.

[9] M.L. Coluccio, G. Das, F. Mecarini, F. Gentile, A. Pujia, L. Bava, R. Tallerico, P. Candeloro, C. Liberale, F. De Angelis, E. DiFabrizio, Microelect. Eng. 86 (2009) 1085-1088.

[10] C.J.L. Constantino, T. Lemma, P.A. Antunes, R. Aroca, Anal. Chem. 73 (2001) 3674-3678.

[11] F. De Angelis, G. Das, P. Candeloro, M. Patrini, M. Galli, A. Bek, M. Lazzarino, I. Maksymov, C. Liberale, L.C. Andreanni, E. DiFabrizio, Nat. Nanotechnol. 5 (2010) 67-72.

[12] F. De Angelis, M. Patrini, G. Das, I. Maksymov, M. Galli, L. Businaro, L.C Andreanni, E. DiFabrizio, Nano Lett. 8 (2008) 2321-2327.

[13] X. Zhang, A.V. Whitney, J. Zhao, E.M. Hicks, R. Van Duyne, J. Nanosci. Nanotech. 6 (2006) 1.

[14] P.N. Barlette, J.J. Baumberg, P.R. Birkin, M.A. Ghanem, M.C. Netti, Chem. Mat. 14 (2002) 2199-2208.

[15] R. Proietti Zaccaria, P. Verma, S. Kawaguchi, S. Shoji, S. Kawata, Opt. Exp. 16 (2008) 14812-14820.

[16] H. Masuda, K. Nishio, Synthesis and applications of highly ordered anodic porous alumina, in: M. Adachi, D.J. Lockwood (Eds.), Self-organized nanoscale materials, Springer publishing Inc., New York, 2006, pp. 296-312.

[17] S. Shingubara, J. Nano. Res. 5 (2003) 17-30.

[18] G.D. Sulka, K.G. Parkola, Thin Sol. Films 515 (2006) 338-345.

[19] E. Dulkeith, A.C. Morteani, T. Niedereichholz, T.A. Klar, J. Feldmann, Phys. Rev. Lett., 89 (2002) 203002(1-4).

20] M. Salerno, N. Patra, R. Cingolani, Nanoscale Res. Lett. 4 (2009) 865-872.

[21] E. Vogel, A. Gbureck, W. Kiefer, J. Mol. Struct. 550-551 (2000) 177-190.

[22] A. Kudelski, Chem Phys. Lett. 414 (2005) 271-275.

[23] C. Murali, S.M. Sharma, S. Karmakar, S.K. Sikka, Physica B 339 (2003) 23-30.

[24] P. Zhang, Y. Zhang, S. Han, Q. Yan, J. Li, Acta Phys. Pol. A 109 (2006) 399-404.

[25] H.J. Lezec, T. Thio, Opt. Exp. 12 (2004) 3629-3651. 